\title{
$Z$ teste e tomada de decisão: Avaliação psicológica ideográfica para manuseio de arma de fogo
}

\author{
$\mathrm{Z}$ test and decision making: Ideographic psychological assessment for firearm handling \\ Prueba $\mathrm{Z}$ y toma de decisiones: Evaluación psicológica ideográfica para el manejo de armas de \\ fuego
}

Recebido: 11/03/2021 | Revisado: 18/03/2021 | Aceito: 23/03/2021 | Publicado: 30/03/2021

Astrid Sharon Pontes de Sá Hasbun
ORCID: http://orcid.org/0000-0002-3757-2841
Universidade Potiguar, Brasil
E-mail: astrid.sharon @ gmail.com
Nilton Soares Formiga
URCID: http://orcid.org/0000-0003-4907-9736
Universidade Potiguar, Brasil
E-mail: nsformiga @ yahoo.com
Ionara Dantas Estevam
ORCID: http://orcid.org/0000-0002-4123-3244
Universidade Potiguar, Brasil

\section{Resumo}

A legislação brasileira atual sobre armamento estabelece normas que restringem o porte de arma no Brasil e torna obrigatória a realização de avaliação psicológica para aquisição e porte de arma de fogo. No Brasil, hoje, embora exista um perfil psicológico pré-definido pela Polícia Federal que orienta o psicólogo em sua tomada de decisão na avaliação psicológica para concessão de registro de arma de fogo, este aspecto carece de maior embasamento teórico. O objetivo deste estudo é traçar indicadores do $\mathrm{Z}$ teste para auxiliar na tomada de decisão nas avaliações psicológicas para manuseio de arma de fogo. A amostra foi composta por 60 sujeitos do sexo masculino de 19 a 51 anos divididos em dois grupos: o G1 com 30 candidatos a obtenção de registro e/ou porte de arma pela Polícia Federal e o G2 com 30 sujeitos que não foram candidatos a obtenção de porte pela Polícia Federal. Foi aplicado o Z teste e utilizado os dados sociodemográficos presentes na folha de administração individual do referido teste. Após a classificação das respostas e análise de cada protocolo foram identificados os indicadores que permitem uma melhor definição dos construtos psicológicos a serem avaliados para manuseio de arma de fogo. Os resultados alcançados demonstraram correlações significativas de confiabilidade do instrumento. Conclui-se que o $\mathrm{Z}$ teste é um instrumento capaz de oferecer indicadores importantes de aptidão ao psicólogo responsável pela concessão do porte de arma de fogo e deve ser incluído na bateria de avaliação psicológica para manuseio de arma de fogo.

Palavras-chave: Avaliação psicológica; Z teste; Indicadores de aptidão; Porte de arma de fogo.

\begin{abstract}
The current Brazilian legislation on weapons establishes norms that restrict the carrying of a weapon in Brazil and makes psychological evaluation mandatory for the acquisition and carrying of a firearm. In Brazil today, although there is a psychological profile pre-defined by the Federal Police that guides the psychologist in his decision making in the psychological evaluation for granting firearm registration, this aspect lacks further theoretical foundation. The objective of this study was to draw indicators of the $\mathrm{Z}$ test to assist in the decision making process in psychological evaluations for handling a firearm. The sample was composed of 60 male subjects between 19 and 51 years of age divided into two groups: G1, with 30 candidates to obtain a gun registration and/or license from the Federal Police, and G2, with 30 subjects who were not candidates to obtain a license from the Federal Police. The $\mathrm{Z}$ test was applied and the sociodemographic data from the individual administration sheet of the test were used. After the classification of the answers and analysis of each protocol, the indicators that allow a better definition of the psychological constructs to be evaluated for firearm handling were identified. The results achieved showed significant correlations of reliability of the instrument. We conclude that the $\mathrm{Z}$ test is an instrument capable of offering important indicators of aptitude to the psychologist responsible for granting a firearm permit and should be included in the psychological evaluation battery for firearm handling.
\end{abstract}

Keywords: Psychological assessment; Z test; Fitness indicators; Firearm possession.

\section{Resumen}

La actual legislación brasileña sobre armas establece normas que restringen el porte de armas de fuego en Brasil y obliga a realizar una evaluación psicológica para la adquisición y posesión de armas de fuego. En Brasil hoy, si bien existe un perfil psicológico predefinido por la Policía Federal que orienta al psicólogo en su toma de decisiones en la evaluación psicológica para la concesión de un registro de arma de fuego, este aspecto carece de un mayor fundamento teórico. El objetivo de este estudio es rastrear indicadores de la prueba $\mathrm{Z}$ para ayudar en la toma de decisiones en evaluaciones psicológicas para el manejo de armas de fuego. La muestra estuvo conformada por 60 sujetos varones de 
19 a 51 años divididos en dos grupos: G1 con 30 candidatos a registro y / o posesión de un arma por la Policía Federal y G2 con 30 sujetos que no fueron candidatos a obtención por la Policía Federal. Se aplicó la prueba Z y se utilizaron los datos sociodemográficos presentes en la hoja de administración individual de esa prueba. Luego de la clasificación de respuestas y análisis de cada protocolo, se identificaron los indicadores que permiten una mejor definición de los constructos psicológicos a evaluar para el manejo de armas de fuego. Los resultados obtenidos mostraron correlaciones significativas de confiabilidad del instrumento. Se concluye que la prueba $\mathrm{Z}$ es un instrumento capaz de ofrecer importantes indicadores de aptitud al psicólogo responsable de otorgar la posesión de un arma de fuego y debe incluirse en la batería de evaluación psicológica para el manejo de armas de fuego.

Palabras clave: Evaluación psicológica; Prueba Z; Indicadores de aptitud; Posesión de armas de fuego.

\section{Introdução}

Nos últimos anos a violência no Brasil tem aumentado de forma exponencial, os veículos de comunicação e redes sociais espalham os acontecimentos em tempo real. A violência é um problema de ordem pública que faz parte da história política do Brasil e que está relacionado à crise da segurança pública, ao crescimento das injustiças sociais e ao esvaziamento de direitos.

Dentre a violência contemporânea, Oliveira, Luna e Silva (2020) sinalizam que os homicídios representam a expressão mais grave de violência e têm um impacto negativo sobre a população, uma vez que é a primeira causa de morte nas camadas jovens da população, superando todas as demais causas. Dessa forma, resultam em medo e insegurança, alterando o comportamento das pessoas, e comprometem a cidadania e os direitos humanos.

Segundo o Atlas da Violência divulgado pelo Instituto de Pesquisa Econômica Aplicada - IPEA (IPEA, 2020), publicação esta que utiliza dados do sistema de informação de mortalidade do Ministério da Saúde e traz informações até 2018, retrata que a taxa de homicídios por arma de fogo no Brasil subiu 15,4\% entre 2008 e 2018 passando de 34.147 para 41.179.

Em virtude do aumento da violência no país, muitas pessoas buscam atualmente a arma de fogo como forma de proteger a si mesmas, suas famílias, seus bens e patrimônios (Resende, 2019). E em consequência dessa realidade a oferta e procura pela obtenção de registro e porte de arma de fogo também tem aumentado no Brasil. Para além dessa realidade existem os indivíduos que fazem uso de armas de fogo em função da sua atividade laborativa como: vigilantes, seguranças, policiais e guardas municipais.

Ao retratar a legislação brasileira da área de armamento, pode-se citar a Lei n ${ }^{\circ} 10.826$, de 23 de dezembro de 2003 , que é um reflexo do aumento da violência nos últimos anos. Esta lei denominada de Estatuto do Desarmamento revoga a Lei $\mathrm{n}^{\circ}$ 9.437/1997 e traz importantes contribuições sobre a regulamentação para obtenção de registro e porte de arma de fogo como: a idade mínima de 25 anos; o porte deixa de ser concedido pelo Ministério do Exército e torna-se responsabilidade da Polícia Federal; o porte ilegal passa a ser considerado um crime inafiançável e fica proibido o porte de arma para qualquer pessoa que não tenha necessidade do uso da arma de fogo para atividade profissional ou que não faça parte de corporações como forças armadas, segurança armada e empresas de segurança (Brasil, 2003).

Nesse cenário, a comprovação de aptidão psicológica, com a finalidade de avaliar a estrutura da personalidade, tornou-se um dos requisitos necessários para os sujeitos que desejam registrar uma arma ou para aqueles que necessitam do porte de arma. O Decreto $n^{\circ}$ 5.123/2004 acrescenta em seu artigo 12, que o interessado em adquirir arma de fogo deve comprovar aptidão psicológica atestada em laudo conclusivo fornecido por psicólogo do quadro da Polícia Federal ou por ela credenciado (Brasil, 2004).

Outra legislação que merece destaque é a Instrução Normativa (IN) nº 78, de 10 de fevereiro de 2014, que estabelece procedimentos para o credenciamento, fiscalização, aplicação e correção dos exames psicológicos realizados por psicólogos credenciados, responsáveis pela expedição do Laudo que ateste aptidão psicológica para o manuseio de arma de fogo. Para realizar a avaliação psicológica, o psicólogo deve ser credenciado pela Polícia Federal, estar inscrito regularmente no Conselho Regional de Psicologia e dominar as técnicas e instrumentos psicológicos utilizados (Brasil, 2014). 
Entretanto, na contramão das pesquisas e evidências científicas que sugerem o controle responsável das armas, desde 2019 tem havido mudanças na legislação brasileira que traz flexibilizações no acesso da população às armas de fogo. Entre janeiro de 2019 e julho de 2020 foram editados, segundo o Atlas de violência (IPEA, 2020), onze decretos, uma lei e quinze portarias do exército que incentivam à disseminação das armas de fogo.

Todavia, ainda segundo o Atlas da violência de 2020, vários desses documentos foram revogados apontando para uma falta de reflexão e embasamento técnico para uma produção adequada de documentos e avaliação de impactos futuros que podem durar décadas (IPEA, 2020).

Segundo a Cartilha de Avalição Psicológica do Conselho Federal de Psicologia (CFP), a capacitação em avaliação psicológica prevê ter conhecimentos dos fundamentos básicos da Psicologia, domínio da psicopatologia, conhecimentos da psicometria e ter domínio dos procedimentos de aplicação, levantamento e interpretação dos instrumentos e técnicas utilizadas na avaliação psicológica, bem como ter condições de planejar a avaliação adequando-a ao objetivo, público-alvo e contexto. Além dessas competências, o psicólogo deve ser capaz de conduzir uma entrevista com o candidato a fim de responder demandas específicas ou realizar devolutiva com o candidato em avaliação (CFP, 2013).

Após a aplicação dos testes, análise dos dados e realização da entrevista o psicólogo precisa tomar a decisão se o sujeito tem ou não condições de manusear/portar uma arma de fogo. No Brasil, hoje, embora exista um perfil psicológico prédefinido pela Polícia Federal que orienta o psicólogo em sua tomada de decisão na avaliação psicológica para concessão de registro de arma de fogo, este aspecto carece de maior embasamento teórico (Resende, 2017).

No que diz respeito aos termos registro, manuseio e porte de arma de fogo é preciso esclarecer que há uma diferença entre eles. Entende-se como manuseio o ato de manusear uma arma, mas este não se estende ao porte que traz o direito de levar a arma consigo de um local a outro. Em outras palavras: todo porte tem um registro de arma e manuseio inerentes, já o registro não comporta em si o porte de arma, apenas o manuseio. Mas qualquer pessoa que manusear uma arma deveria, segundo a legislação, ter um registro ou porte de arma de fogo.

A já citada Instrução $n^{\circ}$ 078/2014 traz a nomenclatura de manuseio de arma de fogo como referência aos processos de aquisição, registro, transferência, renovação de registro e porte de arma (Brasil, 2014). Dessa forma, será usada a nomenclatura de manuseio de arma de fogo como referência neste estudo por entender que o termo é mais amplo e atende às outras nomenclaturas não sendo limitador e tão pouco restritivo além de atender a todos os processos mencionados na referida Instrução.

A escolha do instrumento para esse estudo, o Z -Teste, se deu em função deste ser considerado um teste psicológico projetivo dos mais pertinentes para identificar as características da personalidade que são mais apropriadas para um indivíduo portar ou não uma arma de fogo, bem como por ser um teste que traz vários dos indicadores de aptidão sugeridos pela IN n'78/2014.

De forma geral, o presente estudo tem sua importância por contribuir para a diminuição da lacuna existente de estudos teóricos/científicos na área de avaliação psicológica para manuseio de arma de fogo, trazendo uma contextualização atual sobre o tema e apresentando dados que contribuem para a tomada de decisão por parte do profissional psicólogo.

\section{Metodologia}

\section{Tipo da pesquisa}

Trata-se de uma pesquisa de campo, de natureza quantitativa, do tipo descritiva e exploratória. Especificamente, de acordo com Prodanov (2013) a pesquisa descritiva visa descrever as características de determinada população ou fenômeno ou o estabelecimento de relações entre variáveis e procura descobrir a frequência com que um fato ocorre, sua natureza, suas características, causas, relações com outros fatos. 


\section{Local}

A pesquisa foi realizada no Serviço Integrado de Psicologia (SIP) da Universidade Potiguar em função da possiblidade de utilização de sua estrutura. uma vez que o Mestrado de Psicologia é desenvolvido na mesma unidade em que funciona o SIP, Roberto Freire. Além disso outro fator que contribuiu para essa escolha é que esse estudo tem a participação de graduandos do curso de Psicologia da iniciação científica.

O referido serviço contém salas adequadas para a finalidade de avaliação psicológica, conforme orientado pelo Conselho Federal de Psicologia, ou seja, silencioso, sem estimulação ambiental excessiva, bem iluminado e arejado.

\section{Participantes}

A amostra foi constituída de 60 sujeitos do sexo masculino com idade entre 19 e 51 anos. Os sujeitos foram divididos em 02 grupos: grupo porte de arma: sujeitos que se submeteram a avaliação psicológica para manuseio de arma de fogo; e grupo sem porte de arma: Sujeitos que não se submeteram à avaliação do psicológica para o manuseio de arma de fogo.

No que se refere aos critérios de inclusão, consideraram-se aqueles sujeitos que se submeteram a avaliação para manuseio de arma de fogo (grupo porte de arma); sujeitos que não se submeteram a avaliação para manuseio de arma de fogo e que se dispuseram a participar da pesquisa. Já, em relação aos critérios de exclusão, foram aqueles que não se submeteram à avaliação para o porte de arma e/ou se recusaram a participar da pesquisa, não respondendo o instrumento e com menos de 19 anos ou mais de 51 anos de idade;

\section{Instrumento da pesquisa}

Para a coleta desses dados, foi utilizado o $Z$ teste em sua forma individual e os dados sociodemográficos presentes na folha de administração individual do referido teste.

$\mathrm{O} Z$ teste é um instrumento que avalia a personalidade e foi criado com base metodológica no Psicodiagnóstico de Rorschach. O Z teste é composto por três diapositivos ou pranchas de 18,5 por $25 \mathrm{~cm}$ com manchas de tinta ambíguas, bilaterais e desestruturadas, sendo uma acromática, uma policromática e outra em preto e vermelho. Ao ser exposto às manchas, a tarefa do sujeito é responder "o que isto poderia ser", de modo livre.

As manchas apresentadas nos 03 (três) cartões do $Z$ teste causam nos avaliandos, segundo Vaz e Alchieri (2016), associações percepto-associativas que de alguma forma reproduzem situações internas, seu modo de pensar, de sentir e de tomar decisões. Segundo Villemor-Amaral e Machado (2011), o Z teste examina a estrutura e a dinâmica da personalidade a partir da percepção dos estímulos não estruturados (manchas de tinta) que se apresentam ao sujeito. Essas informações traduzidas nas diversas categorias, determinantes e localizações são quantificadas e interpretadas a partir do dinamismo da personalidade e de sua natureza multifacetada.

Após a fase de associação, o sujeito inicia a fase de inquérito, respondendo onde está aquilo que viu na mancha e o que tem na mancha que lhe fez parecer conforme descrito. Em seguida, o teste é codificado, cada elemento da resposta é classificado por uma série de códigos que determinam e qualificam as respostas, como códigos de forma, cor e movimento, por exemplo. Por fim, a frequência de todos os códigos é computada e interpretada, possibilitando fazer a análise de acordo com as tabelas normativas correspondentes.

No Brasil há três versões do $Z$ teste que possuem o parecer favorável pelo Conselho Federal de Psicologia, o Z-Teste coletivo e individual, validado pelos autores Cícero Vaz e João Carlos Alchieri que utiliza o sistema Klopfer para a interpretação dos resultados; o Teste de Zulliger no Sistema Compreensivo - ZSC - forma individual, dos autores Anna Elisa Villemor-Amaral e Ricardo Primi, que se fundamenta no Sistema Compreensivo desenvolvido por Exner para a aplicação, classificação e interpretação dos dados e o teste de Zulliger no sistema Escola de Paris: forma individual dos autores Elizabeth 
do Nascimento e Marcelo Augusto Resende que se ampara no sistema de correção e interpretação, conhecido inicialmente como "Escola Francesa", idealizado por Ombredane e Canivet.

A técnica utilizada neste estudo é o $Z$ teste sob a perspectiva do Sistema Klopfer, desenvolvido por Bruno Klopfer em 1936 que é um dos modelos norte-americanos que possibilita integração de dados quantitativos e qualitativos. Vaz e Alchieri (2016) sinalizam que Klopfer introduziu alguns elementos básicos diferenciados dos demais sistemas metodológicos como Sombreado de Textura, Cor Simbólica e Movimento Inanimado.

Para a aplicação do $Z$ teste houve a participação de duas estudantes de graduação em Psicologia de iniciação científica da Universidade Potiguar que foram capacitadas pela coordenadora da pesquisa através de um curso de extensão do $Z$ teste com duração de 30 horas entre os meses de setembro e outubro de 2019. O intuito do referido curso foi capacitar as estudantes para a aplicação do $Z$ teste na coleta de dados desta pesquisa. A correção e análise dos dados foi realizada pela coordenadora da Projeto de Pesquisa.

\section{Procedimentos de coleta de dados}

A coleta de dados da pesquisa ocorreu após a aprovação do comitê de ética 5537 - UFRN - Universidade Federal do Rio Grande do Norte - Lagoa Nova Campus Central, através de parecer n ${ }^{\circ}$ 3.948.654. A seleção da amostra dos participantes em cada grupo sobre porte de arma foi através da técnica de amostragem não probabilística, do tipo bola de neve.

Com isso, utilizou-se o pacote estatístico GPower 3.1 para verificar a qualidade da amostra para este estudo. O GPower 3.1 trata-se de um software destinado ao cálculo do poder estatístico (isto é, o teste de hipótese). Este, calcula tanto o 'n' necessário para a pesquisa, quanto, a sua associação ao tipo de cálculo que realizar-se-á (Faul, Erdfelder, Lang, \& Buchner, 2007).

Para a coleta de dados deste estudo, considerando uma probabilidade de $95 \%(\mathrm{p}<0,05)$, magnitude do efeito amostral $(r \geq 0,50)$ e um padrão de poder hipotético $(\pi \geq 0,80)$, observou-se que, a partir desses critérios, uma amostra mínima de 30 respondentes, por grupo, revelou-se ser suficiente para o projeto em questão, tendo os seguintes indicadores da qualidade amostral para ambas as amostras: $t \geq 1,98 ; \pi=0,95 ; \mathrm{p}<0,05$.

Após calculada a proporção necessária da amostra, foram convidados a participar da pesquisa adultos entre 19 e 51 anos do sexo masculino. $\mathrm{O}$ convite à pesquisa foi feito por meio de formulário eletrônico que foi divulgado nos contatos pessoais da equipe de pesquisa (Orientadora, mestranda e estagiárias de iniciação científica), através do aplicativo whatsapp, pela facilidade de acesso a um maior número de pessoas.

Com o sujeito tendo aceitado o convite em participar da pesquisa, era agendado local e hora para aplicação do instrumento. Uma vez que o sujeito preenchia os critérios de inclusão descritos anteriormente, ele assinava o Termo de Consentimento Livre e Esclarecido (TCLE), cujo objetivo é informar aos participantes sobre os procedimentos éticos para a condução da pesquisa e registrar o seu consentimento, de acordo com a resolução do Conselho Nacional de saúde (CNS) nº66/2012, e então tornava-se parte integrante da pesquisa.

Os voluntários foram submetidos a uma aplicação individual, uma vez que esta propicia maiores vantagens sobre a aplicação coletiva, como o contato mais próximo com o examinado, a possibilidade de esclarecer dúvidas e obtenção de inquéritos mais claros e objetivos. Assim como também é possível observar as mudanças de posição dos cartões do teste e reações do sujeito frente aos mesmos.

As aplicações foram realizadas pela equipe de pesquisa, devidamente treinada para a aplicação do $\mathrm{Z}$ teste e a codificação dos protocolos, assim como suas análises foram realizadas pela pesquisadora. Os dados obtidos foram agrupados em tabelas que permitiram as análises estatísticas. 


\section{Tratamento dos dados}

As respostas dos testes foram analisadas a partir da nomenclatura proposta por Vaz e Alchieri (2016), utilizando também, estatística descritivas e inferenciais. Após a classificação das respostas e análise de cada protocolo foram identificados os indicadores que permitem uma melhor definição dos construtos psicológicos a serem avaliados para manuseio de arma de fogo.

Para isso, os dados foram analisados através do pacote estatístico SPSSWIN, em sua versão 24.0 e nele foram tabulados os dados e realizadas as análises estatísticas descritivas e os cálculos referentes a normalidade da amostra e Anova One-way, associada ao teste post-hoc de Scheffé para verificar o efeito significativo das diferenças entre os grupos, e o TDE (Tamanho Do Efeito), acompanhado pelo 'd' de Cohen, responsáveis para avaliar a dimensão com que o fenômeno estaria presente na população ou amostra pesquisada, sendo assim, exclusivo desta.

\section{Resultados}

Finalizada a coleta de dados, a qual, ocorreu no período de 30 de outubro de 2019 a 01 de março de 2020, para ambas as amostras e seus contextos de coleta por meio físico, no Serviço Integrado de Psicologia da Universidade Potiguar, na cidade do Natal-RN.

Tendo o tamanho da amostra sido alcançado para os dois grupos, as respostas de cada participante foram inseridas no programa Excell e, em seguida, transferidas para o pacote estatístico SPSS 24.0, no qual, foram realizadas as análises estatísticas.

Com isso, numa primeira etapa de conferência dos resultados, verificou-se que não houve respostas duplicadas e/ou missing (itens sem respostas), a fim de verificar se existiria algum erro de preenchimento por parte dos respondentes, o qual foi identificado ambos os grupos concluíram toda a tarefa apresentada pela pesquisadora aos participantes.

Desta forma, no que se refere a multicolinearidade entre as variáveis, observou-se a existência de correlações que variaram de 0,09 a 0,42; sendo assim, (r) $\leq 0,90$, de acordo com o que estabeleceu Tabachnick e Fidell (2001). Ao considerar esse resultado, é possível afirmar o baixo grau de correlação, condição a qual, não interfere no desenvolvimento e elaboração de hipótese e modelos com erro de medidas.

No que diz respeito aos outliers multivariados, realizou-se o teste de normalidade de Shapiro-Wilk (S-W), utilizada para análise de amostras inferiores a 100 sujeitos (Nascimento et al., 2014). Desta forma, observou-se tanto para a amostra total (60 sujeitos), quanto para a distribuição amostral em ambos os grupos ( $\mathrm{G} 1=30$ e G2 $=30$ ), a existência de uma normalidade ( $\mathrm{S}-\mathrm{W}=0,97 ; \mathrm{P}<0,56)$.

Verificou-se também, a tendência de não resposta a pesquisa, utilizando o procedimento de Armstrong e Overton (1977) (Armstrong \& Overton, 1977). Este, por sua vez, avalia as diferenças entre os primeiros $(\mathrm{n} 1=10)$ e os últimos $(\mathrm{n} 2=$ 10) participantes da amostra de uma pesquisa, para a qual considerou-se dez (10) sujeitos iniciais e finais para grupo ( G1 e G2) de participantes.

A partir do teste de Levene, verificou-se a igualdade das variâncias e com um teste $t$ de Student avaliou-se a igualdade dos meios. Observou-se que tal diferença não foi significativa (isto é, $\mathrm{p}<0,19$ ) entre os escores médios nas amostras coletadas ( com $\mathrm{t} \leq 1,96$ ). Desta maneira, ao considerar tais resultados, não existe viés de não resposta, não sendo com isso, um problema significativo para à avaliação das respostas e verificação das hipóteses.

A partir da qualificação das amostras e que elas não representam problemas para as análises, pois, com base nos critérios estatísticos assumidos, elas atendem a normalidade, podendo realizar os cálculos paramétricos destinados à avaliação da variância dos escores médios entre as variáveis, procurando atender ao objetivo central desse estudo; a título de lembrança 
para o leitor: verificar as diferenças entre os escores médios para as pessoas que submeteram e não submeteram à avaliação psicológica pelo porte de armas.

Com isso, avaliaram-se as características sociodemográficas desse estudo, observando que: há uma distribuição equitativa entre as duas amostras, as quais, apresentaram 30 participantes por grupo, 100\% da amostra é composta por homens, com idade de 19 a 51 anos (Média $=41,30$, d.p. = 14,91), 67\% tem curso superior e no que diz respeito às características profissionais destes, em termos de uma hierarquia de frequências, observou-se que $31 \%$ são assistentes administrativos, $13 \%$ aposentados, $7 \%$ empresários, $6 \%$ agentes (ambientais ou penitenciários), $5 \%$ comerciantes, $5 \%$ estudantes, $5 \%$ professores. As demais profissões não atingiram 5\% da amostra, o que equivale menos de 3 participantes da pesquisa.

A partir desses resultados, realizou-se o cálculo da Anova one-way, associada ao teste post-hoc de Scheffé, destinados à avaliação dos Determinantes no $\mathrm{Z}$ teste, os quais, revelaram os seguintes escores médios: na tabela 1 podemos verificar os escores médios entre os grupos em função dos determinantes e localização das respostas no $\mathrm{Z}$ teste. Observou-se que no FORMPREC (Forma precisa), MOVIANI (Movimento Animal), CONTANIM (Conteúdo Animal) e DETALCOMU (Localização de respostas detalhe comum), o grupo que não participou da avaliação para o porte de arma apresentou maiores escores do que o grupo que fez avaliação para o porte de arma (respectivamente, $\mathrm{F}_{1 / 60}=5,54$, $\mathrm{p}<0,01 ; \mathrm{F}_{1 / 60}=9,47$, $\mathrm{p}<0,01$; $\left.\mathrm{F}_{1 / 60}=22,93, \mathrm{p}<0,01 ; \mathrm{F}_{1 / 60}=4,61, \mathrm{p}<0,01\right)$.

Por outro lado, ainda na tabela 1, na CORFOM (Cor e Forma) e CORPURA (Cor pura), os maiores escores estiveram para os participantes da avaliação psicológica destinado ao porte de arma (respectivamente, $\mathrm{F}_{1 / 60}=7,59, \mathrm{p}<0,01 ; \mathrm{F}_{1 / 60}=6,67$, $\mathrm{p}<0,01)$. Os resultados expressos nestes parágrafos, revelam quando se avaliou o teste de Scheffé, que os escores médios, foram significativos.

Um resultado adicional para estas variáveis, apenas aquelas significativas, pode destacar, ao avaliar os seus escores totais que, hierarquicamente, apresentaram escores mais altos na seguinte ordem: DETALCOMU, CONTANIM, FORMPREC, MOVIANI, CORFOM e CORPURA. 
Tabela 1. Escores médios entre os grupos em função dos determinantes e localização das respostas no $\mathrm{Z}$ teste.

\begin{tabular}{|c|c|c|c|c|c|c|c|c|c|}
\hline \multirow[b]{2}{*}{ Variáveis } & \multirow[b]{2}{*}{ Grupos } & \multirow[b]{2}{*}{$\mathrm{N}$} & \multirow[b]{2}{*}{ Média } & \multirow[b]{2}{*}{ d.p. } & \multicolumn{2}{|c|}{$95 \%$ IC } & \multicolumn{3}{|c|}{ Estatística } \\
\hline & & & & & Baixo & Alto & $\begin{array}{c}\mathrm{F} \\
\text { (Friedman) }\end{array}$ & $\mathrm{gl}$ & p-valor \\
\hline \multicolumn{10}{|l|}{$\begin{array}{l}\text { Variáveis Z teste - } \\
\text { Determinantes }\end{array}$} \\
\hline \multirow{3}{*}{ FORMA } & G1 Porte & 30 & 3,67 & 2,37 & 2,77 & 4,56 & \multirow{3}{*}{3,02} & \multirow{3}{*}{1} & \multirow{3}{*}{0,09} \\
\hline & $\begin{array}{l}\text { G2 Não } \\
\text { porte }\end{array}$ & 30 & 4,80 & 2,44 & 3,81 & 5,79 & & & \\
\hline & Total & 60 & 4,23 & 2,67 & 3,57 & 4,90 & & & \\
\hline \multirow{3}{*}{ FORMPREC } & G1 Porte & 30 & 2,27 & 1,56 & 1,57 & 2,96 & \multirow{3}{*}{5,54} & \multirow{3}{*}{1} & \multirow{3}{*}{0,01} \\
\hline & $\begin{array}{l}\text { G2 Não } \\
\text { porte }\end{array}$ & 30 & 3,57 & 2,38 & 2,67 & 4,46 & & & \\
\hline & Total & 60 & 2,92 & 2,19 & 2,34 & 3,49 & & & \\
\hline \multirow{3}{*}{ MOVIHUM } & \begin{tabular}{|l|} 
G1 Porte \\
\end{tabular} & 30 & 0,83 & 0,83 & 0,52 & 1,14 & \multirow{3}{*}{1,42} & \multirow{3}{*}{1} & \multirow{3}{*}{0,24} \\
\hline & $\begin{array}{l}\text { G2 Não } \\
\text { porte }\end{array}$ & 30 & 0,60 & 0,65 & 0,35 & 0,85 & & & \\
\hline & Total & 60 & 0,72 & 0,61 & 0,52 & 0,91 & & & \\
\hline \multirow{3}{*}{ MOVIANI } & G1 Porte & 30 & 0,47 & 0,29 & 0,23 & 0,70 & \multirow{3}{*}{9,47} & \multirow{3}{*}{1} & \\
\hline & $\begin{array}{l}\text { G2 Não } \\
\text { porte }\end{array}$ & 30 & 1,37 & 1,74 & 0,82 & 1,92 & & & 0,01 \\
\hline & Total & 60 & 0,92 & 1,11 & 0,60 & 1,23 & & & \\
\hline & G1 Porte & 30 & 0,87 & 0,90 & 0,53 & 1,20 & & & \\
\hline FORMCOR & $\begin{array}{l}\text { G2 Não } \\
\text { porte }\end{array}$ & 30 & 1,07 & 1,37 & 0,57 & 1,57 & 0,46 & 1 & 0,50 \\
\hline & Total & 60 & 0,97 & 1,13 & 0,67 & 1,26 & & & \\
\hline & G1 Porte & 30 & 0,43 & 0,77 & 0,14 & 0,72 & & & \\
\hline CORFORM & $\begin{array}{l}\text { G2 Não } \\
\text { porte }\end{array}$ & 30 & 0,03 & 0,18 & $-0,03$ & 0,10 & 7,59 & 1 & 0,01 \\
\hline & Total & 60 & 0,23 & 0,59 & 0,08 & 0,39 & & & \\
\hline & G1 Porte & 30 & 0,63 & 0,99 & 0,26 & 1,01 & & & \\
\hline SOMBPERSC & \begin{tabular}{|l} 
G2 Não \\
porte
\end{tabular} & 30 & 0,50 & 0,82 & 0,19 & 0,81 & 0,32 & 1 & 0,57 \\
\hline & Total & 60 & 0,57 & 0,90 & 0,33 & 0,80 & & & \\
\hline & G1 Porte & 30 & 0,10 & 0,30 & $-0,01$ & 0,21 & & & \\
\hline SOMPERSDISF & $\begin{array}{l}\text { G2 Não } \\
\text { porte }\end{array}$ & 30 & 0,00 & 0,00 & 0,00 & 0,00 & 3,22 & 1 & 0,08 \\
\hline & Total & 60 & 0,05 & 0,22 & $-0,01$ & 0,11 & & & \\
\hline & G1 Porte & 30 & 0,50 & 0,68 & 0,25 & 0,75 & & & \\
\hline CORACROM & $\begin{array}{l}\text { G2 Não } \\
\text { porte }\end{array}$ & 30 & 0,43 & 0,62 & 0,18 & 0,69 & 0,14 & 1 & 0,71 \\
\hline & Total & 60 & 0,47 & 0,67 & 0,29 & 0,64 & & & \\
\hline & G1 Porte & 30 & 0,10 & 0,40 & $-0,05$ & 0,25 & & & \\
\hline TEXTURA & $\begin{array}{l}\text { G2 Não } \\
\text { porte }\end{array}$ & 30 & 0,13 & 0,43 & $-0,03$ & 0,30 & 0,10 & 1 & 0,75 \\
\hline & Total & 60 & 0,12 & 0,41 & 0,01 & 0,22 & & & \\
\hline & G1 Porte & 30 & 0,30 & 0,53 & 0,10 & 0,50 & & & \\
\hline CORPURA & $\begin{array}{l}\text { G2 Não } \\
\text { porte }\end{array}$ & 30 & 0,03 & 0,18 & $-0,03$ & 0,10 & 6,67 & 1 & 0,01 \\
\hline & Total & 60 & 0,17 & 0,41 & 0,06 & 0,27 & & & \\
\hline & G1 Porte & 30 & 1,77 & 1,25 & 1,30 & 2,23 & & & \\
\hline CONTHUM & $\begin{array}{l}\text { G2 Não } \\
\text { porte }\end{array}$ & 30 & 1,33 & 0,88 & 1,00 & 1,66 & 2,40 & 1 & 0,13 \\
\hline & Total & 60 & 1,55 & 1,09 & 1,27 & 1,83 & & & \\
\hline & G1 Porte & 30 & 2,27 & 1,36 & 1,76 & 2,78 & & & \\
\hline CONTANIM & $\begin{array}{l}\text { G2 Não } \\
\text { porte }\end{array}$ & 30 & 4,67 & 2,33 & 3,78 & 5,56 & 22,93 & 1 & 0,01 \\
\hline & Total & 60 & 3,47 & 2,27 & 2,88 & 4,05 & & & \\
\hline
\end{tabular}




\begin{tabular}{|c|c|c|c|c|c|c|c|c|c|}
\hline \multicolumn{10}{|c|}{$\begin{array}{l}\text { Variáveis Z teste - } \\
\text { Localização das respostas }\end{array}$} \\
\hline \multirow{3}{*}{ GLOBAL } & \begin{tabular}{|l|} 
G1 Porte \\
\end{tabular} & 30 & 1,53 & 1,25 & 1,07 & 2,00 & \multirow{3}{*}{0,26} & \multirow{3}{*}{1} & \multirow{3}{*}{0,61} \\
\hline & $\begin{array}{l}\text { G2 Não } \\
\text { porte }\end{array}$ & 30 & 1,70 & 1,29 & 1,22 & 2,18 & & & \\
\hline & Total & 60 & 1,62 & 1,26 & 1,29 & 1,94 & & & \\
\hline \multirow{3}{*}{ DETALCOMU } & \begin{tabular}{|l|} 
G1 Porte \\
\end{tabular} & 30 & 4,77 & 2,47 & 3,84 & 5,69 & \multirow{3}{*}{4,61} & \multirow{3}{*}{1} & \multirow{3}{*}{0,05} \\
\hline & $\begin{array}{l}\text { G2 Não } \\
\text { porte }\end{array}$ & 30 & 6,17 & 2,57 & 5,21 & 7,13 & & & \\
\hline & Total & 60 & 5,47 & 2,60 & 4,79 & 6,14 & & & \\
\hline \multirow{3}{*}{ DETINCOM } & \begin{tabular}{|l|} 
G1 Porte \\
\end{tabular} & 30 & 0,70 & 0,87 & 0,37 & 1,03 & \multirow{3}{*}{2,76} & \multirow{3}{*}{1} & \multirow{3}{*}{0,10} \\
\hline & $\begin{array}{l}\text { G2 Não } \\
\text { porte }\end{array}$ & 30 & 1,17 & 1,26 & 0,70 & 1,64 & & & \\
\hline & Total & 60 & 0,93 & 1,10 & 0,65 & 1,22 & & & \\
\hline \multirow{3}{*}{ ESPBRAN } & \begin{tabular}{|l|} 
G1 Porte \\
\end{tabular} & 30 & 0,80 & 0,48 & 0,62 & 0,98 & \multirow{3}{*}{0,41} & \multirow{3}{*}{1} & \multirow{3}{*}{0,53} \\
\hline & $\begin{array}{l}\text { G2 Não } \\
\text { porte }\end{array}$ & 30 & 0,90 & 0,71 & 0,63 & 1,17 & & & \\
\hline & Total & 60 & 0,85 & 0,60 & 0,69 & 1,01 & & & \\
\hline
\end{tabular}

Notas: FORMA - Controle Geral, dimensão racional, expressão lógica e intelectual da percepção; FORMPREC- Forma precisa. Funcionamento do pensamento lógico, Capacidade e discriminação perceptiva; MOVHUM - Movimento Humano - Espontaneidade, criatividade, empatia; MOVANI Movimento Animal - Impulsos, dinamismo; FORMCOR- Forma e Cor - Relacionamento Interpessoal. Capacidade de receber e retribuir afeto de forma adequada; CORFORM - Cor e Forma - Indicativo de excitabilidade emocional. Reage de forma precária aos estímulos emocionais. Escapes agressivos e atitudes sem o adequado controle; SOMPERSP - Sombreado perspectiva - Capacidade de adaptação afetiva, cautelosa para melhor suportar o sofrimento e evitar a depressão; SOMBDIS - Sombreado perspectiva disfórico - Ansiedade sem o adequado controle. Condições internas precárias de suportar fortes pressões externas; CORACROM - Cor acromática - Traços depressivos de personalidade; TEXTURA - Textura - Contato humano e interação social; CORPURA - Cor pura - Tendência a reação emocional intensa. Projeção das reações emocionais livres, intensas e carregadas de sentimentos, seja de apreciação, estima, amor, ódio, raiva e agressividade. Ocorre com pouca frequência. Descontrole das emoções, escapes agressivos, instabilidade; CONTHUM - Conteúdo Humano - Modo como a pessoa percebe as outras, sendo assim maior ou menor capacidade de relacionamento; CONTANIM - Conteúdo Animal - Reminiscências do mundo infantil. O que ficou do mundo infantil no adulto. GLOBAL - Localização de respostas Global - Percepção de síntese e capacidade de planejamento; DETALCOMU - Localização de respostas detalhe comum - Visão objetiva da realidade, uso da inteligência voltada para o concreto e pragmático. Percepção da realidade objetiva; DETINCOM - Localização de respostas detalhe incomum - Capacidade de análise, observação, bom senso; ESPBRAN - Presença de Espaço em Branco. Indicador de ansiedade situacional. Expressa sentimento de insegurança, desprazer e tensão diante de dificuldades circunstancial a ser transposta pelo indivíduo.

Fonte: Autores.

No que se refere as variáveis que contemplam questões relativas à categoria tipo de ressonância interna é possível perceber na Tabela 2 os escores médio dos grupos na avaliação do porte de arma em função do tipo de vivência interna. (Tipo de vivência). Observou-se resultados significativos, apenas na RESSEXTR (refere ao sujeito Extratensivo com facilidade para se expor e propensão a perder o controle), na qual, o grupo que realizou a avaliação psicológica apresentou maior escore ao comparar com aqueles que não fizeram avaliação $\left(\mathrm{F}_{1 / 60}=3,44 \mathrm{p}<0,01\right)$ e na variável RESSSCOARTA (diz respeito ao sujeito Coartado. Condições adequadas para lidar com suas condições emocionais. Pode ser considerado o "tipo ideal" - por apresentar equilíbrio entre mundo interno e externo), com os participantes que não fizeram avaliação psicológica para o porte de arma apresentando maiores escores $\left(\mathrm{F}_{1 / 60}=6,93, \mathrm{p}<0,01\right)$ (ver tabela 2).

É preciso destacar, que para tais resultados, avaliou-se o 'd' de Cohen; o interesse neste tipo de cálculo refere-se a segurança para a objetividade dos resultados entre ambos os grupos e a sua significância. Sendo assim, voltou-se para a análise dos tamanhos do efeito (TDE), a qual, realizou-se através do 'd' de Cohen.

A importância desse indicador se deve ao observar que os testes estatísticos têm seu TDE específico e o índice de TDE, além de apresentar uma vantagem de não dependência do tamanho da amostra, sendo capaz de informar sobre o significado dos resultados e a qualidade métrica para comparação dos resultados em estudos distintos (por exemplo, metaanálise, experimental e/ou quase-experimental e comparação gradual) (Cumming, 2012; Lenhard, \& Lenhard, 2016).

Assim, de acordo com Cohen (1991a;1992b), o TDE poderá ser compreendido em termos do grau ou dimensão em que o fenômeno está presente na população ou amostra pesquisada, sendo exclusivo desta. O poder do teste por sua vez se refere a suposição de probabilidade do pesquisador em relação ao poder de detecção do teste em sinalizar que a diferença observada na comparação entre os grupos ou eventos avaliados, para poucas variáveis ou sujeitos, observou-se uma significância. 
Desta maneira, ainda na tabela 2, pode-se perceber que o 'd' de Cohen, nas variáveis RESSEXTR (Extratensivo. Facilidade para se expor. Propensão a perder o controle. Pode demorar a se dar conta que está fantasiando) e RESSSCOARTA (Coartado. Condições adequadas para lidar com suas condições emocionais. Pode ser considerado o "tipo ideal" - equilíbrio entre mundo interno e externo), variaram de $22 \%$ a $33 \%$, respectivamente.

Apesar destes percentuais revelarem a possibilidade de que a avaliação proposta destinada ao porte de arma com base na estrutura psicológica (especialmente, no que diz respeito a personalidade) destes sujeitos, não é possível afirmar de forma consistente a sua significância, pois, a esta pode-se atribuir uma falsa-verdade, condição a qual, rejeita-se o erro tipo II, firmando uma necessidade parcimoniosa quando se pretender salientar a intervenção avaliativa quanto um efeito real nos grupos avaliados.

Realizou-se também, a comparação destas variáveis em função da idade. Na tabela 03 é possível verificar o escore médio na avaliação do porte de arma em relação da faixa etária em função do tipo de vivência interna. Para isso optou-se em distribuir esta última variável em tercil ( $1=$ adultos jovens-adultos [19 a 31 anos], $2=$ adultos [ 32 a 45 anos] e $3=$ adultos mais velhos [46 a 59 anos]. Sendo assim, em relação ao tipo de ressonância interna, no que se refere a categoria etária, apenas na variável, RESSSCOARTA (diz respeito ao sujeito Coartado. Condições adequadas para lidar com suas condições emocionais. Pode ser considerado o "tipo ideal" - equilíbrio entre mundo interno e externo), os sujeitos mais velhos, com idades de 46 a 83 anos, apresentaram escores maiores do que os demais grupos etários $\left(\mathrm{F}_{2 / 60}=3,40 \mathrm{p}<0,05\right)$. 
Tabela 2. Escore médio dos grupos na avaliação do porte de arma em função do tipo de vivência interna.

\begin{tabular}{|c|c|c|c|c|c|c|c|c|c|c|c|}
\hline \multirow[t]{2}{*}{ Variáveis } & \multirow[t]{2}{*}{ Grupos } & \multirow[t]{2}{*}{$\mathrm{N}$} & \multirow[t]{2}{*}{ Média } & \multirow[t]{2}{*}{ d.p. } & \multicolumn{2}{|c|}{$95 \%$ IC } & \multicolumn{3}{|c|}{ Estatística } & \multirow{2}{*}{$\begin{array}{c}\mathrm{d} \\
\text { Cohen }\end{array}$} & \multirow[t]{2}{*}{ TDE } \\
\hline & & & & & Baixo & Alto & $\begin{array}{c}\mathrm{F} \\
\text { (Friedman) }\end{array}$ & $\mathrm{gl}$ & p-valor & & \\
\hline \multirow{3}{*}{ Ressintro } & G1 Porte & 30 & $\mathbf{0 , 4 3}$ & 0,504 & 0,25 & 0,62 & \multirow{3}{*}{0,07} & \multirow{3}{*}{1} & \multirow{3}{*}{0,80} & \multirow{3}{*}{0,03} & \multirow{3}{*}{$0,1 \%$} \\
\hline & G2 Não porte & 30 & 0,40 & 0,498 & 0,21 & 0,59 & & & & & \\
\hline & Total & 60 & 0,42 & 0,497 & 0,29 & 0,55 & & & & & \\
\hline \multirow{3}{*}{ Ressextr } & G1 Porte & 30 & $\mathbf{0 , 5 3}$ & 0,507 & 0,34 & 0,72 & \multirow{3}{*}{3,44} & \multirow{3}{*}{1} & \multirow{3}{*}{0,05} & \multirow{3}{*}{0,45} & \multirow{3}{*}{$22 \%$} \\
\hline & G2 Não porte & 30 & 0,30 & 0,466 & 0,13 & 0,47 & & & & & \\
\hline & Total & 60 & 0,42 & 0,497 & 0,29 & 0,55 & & & & & \\
\hline \multirow{3}{*}{ Ressamb } & G1 Porte & 30 & 0,00 & 0,000 & 0,00 & 0,00 & \multirow{3}{*}{0,00} & \multirow{3}{*}{1} & \multirow{3}{*}{--- } & \multirow{3}{*}{--- } & \multirow{3}{*}{---} \\
\hline & G2 Não porte & 30 & 0,00 & 0,000 & 0,00 & 0,00 & & & & & \\
\hline & Total & 60 & 0,00 & 0,000 & 0,00 & 0,00 & & & & & \\
\hline \multirow{3}{*}{ Resscoarti } & G1 Porte & 30 & 0,00 & 0,000 & 0,00 & 0,00 & \multirow{3}{*}{1,00} & \multirow{3}{*}{1} & \multirow{3}{*}{0,32} & \multirow{3}{*}{0,23} & \multirow{3}{*}{$11 \%$} \\
\hline & G2 Não porte & 30 & $\mathbf{0 , 0 3}$ & 0,183 & $-0,03$ & 0,10 & & & & & \\
\hline & Total & 60 & 0,02 & 0,129 & $-0,02$ & 0,05 & & & & & \\
\hline \multirow{3}{*}{ Resscoarta } & G1 Porte & 30 & 0,03 & 0,183 & $-0,03$ & 0,10 & \multirow{3}{*}{6,93} & & & & \\
\hline & G2 Não porte & 30 & $\mathbf{0 , 2 7}$ & 0,450 & 0,10 & 0,43 & & 1 & 0,01 & 0,70 & $33 \%$ \\
\hline & Total & 60 & 0,15 & 0,360 & 0,06 & 0,24 & & & & & \\
\hline
\end{tabular}

Nota: RESSINTRO - Introversivo. Afetividade intensa. Criatividade. Instropecção elaborativa. Preocupação com a realidade.; RESSEXTR - Extratensivo. Facilidade para se expor. Propensão a perder o controle. Pode demorar a se dar conta que está fantasiando; RESSAMB - Ambigual. Estreitamento afetivo. Indecisão diante de uma situação de pressão ou conflito; RESSCOARTI - Coartativo. Defensividade, propensão à repressão; RESSACOARTA - Coartado. Condições adequadas para lidar com suas condições emocionais. Pode ser considerado o "tipo ideal" - equilíbrio entre mundo interno e externo.

Fonte: Autores 
Research, Society and Development, v. 10, n. 4, e5910413815, 2021

(CC BY 4.0) | ISSN 2525-3409 | DOI: http://dx.doi.org/10.33448/rsd-v10i4.13815

Tabela 3. Escore médio na avaliação do porte de arma em relação da faixa etária em função do tipo de vivência interna.

\begin{tabular}{|c|c|c|c|c|c|c|c|c|c|}
\hline \multirow[t]{2}{*}{ Variáveis } & \multirow{2}{*}{$\begin{array}{l}\text { Faixa } \\
\text { etária }\end{array}$} & \multirow[t]{2}{*}{$\mathrm{N}$} & \multirow[t]{2}{*}{ Média } & \multirow[t]{2}{*}{ d.p. } & \multicolumn{2}{|c|}{$95 \% \mathrm{IC}$} & \multicolumn{3}{|c|}{ Estatística } \\
\hline & & & & & Baixo & Alto & $\begin{array}{c}\mathrm{F} \\
\text { (Friedman) }\end{array}$ & $\mathrm{gl}$ & p-valor \\
\hline \multirow{4}{*}{ RESSINTRO } & \begin{tabular}{|l} 
19-31anos \\
\end{tabular} & 19 & 0,47 & 0,513 & 0,23 & 0,72 & \multirow{4}{*}{0,46} & \multirow{4}{*}{2} & \multirow{4}{*}{0,64} \\
\hline & 32-45anos & 20 & 0,45 & 0,510 & 0,21 & 0,69 & & & \\
\hline & 46-59anos & 21 & 0,33 & 0,483 & 0,11 & 0,55 & & & \\
\hline & Total & 60 & 0,42 & 0,497 & 0,29 & 0,55 & & & \\
\hline \multirow{4}{*}{ RESSEXTR } & 19-31anos & 19 & 0,53 & 0,513 & 0,28 & 0,77 & \multirow{4}{*}{0,76} & \multirow{4}{*}{2} & \multirow{4}{*}{0,47} \\
\hline & 32-45anos & 20 & 0,40 & 0,503 & 0,16 & 0,64 & & & \\
\hline & 46-59anos & 21 & 0,33 & 0,483 & 0,11 & 0,55 & & & \\
\hline & Total & 60 & 0,42 & 0,497 & 0,29 & 0,55 & & & \\
\hline \multirow{4}{*}{ RESSAMB } & 19-31anos & 19 & 0,00 & 0,000 & 0,00 & 0,00 & \multirow{4}{*}{---} & \multirow{4}{*}{---} & \multirow{4}{*}{---} \\
\hline & 32-45anos & 20 & 0,00 & 0,000 & 0,00 & 0,00 & & & \\
\hline & 46-59anos & 21 & 0,00 & 0,000 & 0,00 & 0,00 & & & \\
\hline & Total & 60 & 0,00 & 0,000 & 0,00 & 0,00 & & & \\
\hline \multirow{4}{*}{ RESSCOARTI } & 19-31anos & 19 & 0,00 & 0,000 & 0,00 & 0,00 & \multirow{4}{*}{0,92} & \multirow{4}{*}{2} & \multirow{4}{*}{0,40} \\
\hline & 32-45anos & 20 & 0,00 & 0,000 & 0,00 & 0,00 & & & \\
\hline & \begin{tabular}{|l}
$46-59$ anos \\
\end{tabular} & 21 & 0,05 & 0,218 & $-0,05$ & 0,15 & & & \\
\hline & Total & 60 & 0,02 & 0,129 & $-0,02$ & 0,05 & & & \\
\hline \multirow{4}{*}{ RESSCOARTA } & 19-31anos & 19 & 0,00 & 0,000 & 0,00 & 0,00 & \multirow{4}{*}{3,40} & \multirow{4}{*}{2} & \multirow{4}{*}{0,05} \\
\hline & 32-45anos & 20 & 0,15 & 0,366 & $-0,02$ & 0,32 & & & \\
\hline & 46-51anos & 21 & 0,29 & 0,463 & 0,08 & 0,50 & & & \\
\hline & Total & 60 & 0,15 & 0,360 & 0,06 & 0,24 & & & \\
\hline
\end{tabular}

Nota: RESSINTRO - Introversivo. Afetividade intensa. Criatividade. Instropecção elaborativa. Preocupação com a realidade.; RESSEXTR - Extratensivo. Facilidade para se expor. Propensão a perder o controle. Pode demorar a se dar conta que está fantasiando; RESSAMB - Ambigual. Estreitamento afetivo. Indecisão diante de uma situação de pressão ou conflito; RESSCOARTI - Coartativo. Defensividade, propensão à repressão; RESSACOARTA - Coartado. Condições adequadas para lidar com suas condições emocionais. Pode ser considerado o "tipo ideal" - equilíbrio entre mundo interno e externo.

Fonte: Autores. 


\section{Discussão}

Considerando os achados desse estudo, foi possível perceber, na tabela 01, maiores escores de forma precisa e Detalhe comum (D) no grupo G2 (não porte) do que no grupo G1 (porte). A forma precisa (F+) está relacionada clareza, precisão, coerência e objetividade, diferenciando pessoas, situações e coisas pelas suas peculiaridades e características. Já a resposta de detalhe comum mostra uma percepção da realidade objetiva, uma expressão do concreto. É possível dessa forma perceber que o G2 se mostra com características de uma inteligência prática, objetiva e concreta.

Da mesma forma observou-se maiores escores no G2 relacionados a movimento animal (FM) e conteúdo animal (Cont. Anim), tabela 01, que trazem relação com características da infância, o que ficou de reminiscências do mundo infantil no adulto. As histórias para crianças estão muitas vezes ligadas a figuras de animais. Os programas infantis e de televisão reforçam isso diariamente.

Vaz e Alchieri (2016) afirmam que um índice elevado de respostas com conteúdo animal pode retratar indivíduos com capacidade de competição e iniciativa, mas pode também estar associado a uma válvula de escape ou ainda uma expressão de experiências vividas no passado, entretanto com um funcionamento mais ajustado à realidade e maduro. Que neste caso específico por estar associado ao conteúdo animal pode significar uma fuga da realidade.

Referente ao G1 (porte de arma) os escores de Cor e Forma (CF) e cor pura (C) foram mais elevados se comparados aos resultados do G2, tabela 01. Esses determinantes estão ligados a indicativos de excitabilidade emocional e descontrole das emoções com escapes agressivos. O determinante Cor e Forma (CF) sugere indivíduos que reagem aos estímulos emocionais de forma precariamente controlada. Para Vaz e Alchieri (2016) seu sistema emocional se mobiliza com a mínima intensidade de estímulo, por isso a tendência a escapes agressivos e atitudes inadequadas. Já a Cor Pura (C) representa a liberação de emoção sem controle.

Para Vaz e Alchieri (2016) as cores constituem um dos aspectos mais importantes do Z teste, pois são estímulos de impacto do mundo externo com poderes de mobilizar o mundo interno do sujeito provocando reações emocionais que podem se apresentar de forma precária quando a mobilização é intensa para o indivíduo.

A partir desse resultado é possível perceber que no grupo de sujeitos que se submeteram à avaliação para porte de arma esse construto de personalidade se revela como comum ao grupo. Podemos então inferir que sujeitos que buscam realizar a avaliação psicológica para manuseio de arma de fogo tendem a apresentar vulnerabilidade emocional aumentada. Este resultado, neste grupo especificamente, é revelador e fala da importância da aplicação desse instrumento neste contexto de avaliação psicológica.

Ainda em relação a relação de $\mathrm{CF}+\mathrm{C}$, os dados encontrados reforçam que a relação $\mathrm{FC}>\mathrm{CF}+\mathrm{C}$ deve ser um indicativo analisado em avaliações para manuseio de arma de fogo. Esta relação aponta que esta razão indica que o examinando é capaz de liberar seus afetos e emoções de forma adequada e madura. Já a relação inversa $(\mathrm{C}+\mathrm{CF}>\mathrm{FC})$ encontrada nos resultados do G1 traz a indicação de reação aos estímulos emocionais de forma precariamente controlada, com tendência a excitabilidade emocional e atitudes sem o adequado controle.

Dando continuidade à análise dos resultados, a ressonância interna é uma das dimensões mais importantes no construto multifacetado que é a personalidade. O tipo de ressonância interna retrata a ressonância dos estímulos cromáticos ( $\Sigma \mathrm{C}$ - somatório de cor), ambiente na dimensão do mundo interno ( $\Sigma \mathrm{M}$ - somatório de movimento humano) na personalidade. Em outras palavras, é o mundo interno em ação.

No que se refere ao tipo de ressonância interna, tabela 02, no G1 (porte de arma) surgiu como escore maior o “extratensivo" que indica facilidade para se expor e propensão a perder o controle. Já o G2 (não porte de arma) apresentou 
como maior escore no tipo de ressonância "Coartado" que sugere condições adequadas para lidar com as condições emocionais. O Coartado pode ser considerado como o "tipo ideal" por apresentar equilíbrio entre o mundo interno e externo.

Nesse momento é importante salientar que os tipos extratensivo e intratensivo não são os introvertidos e extrovertidos de Jung. Vaz e Alchieri (2016) sinalizam que os tipos extratensivo, intratensivo, coartado e coartativo não são características fixas e contínuas da personalidade, mas uma capacidade móvel e não estável na vida podendo intensificar um ou outro dependendo da fase da vida em que o indivíduo se encontra. Crianças tendem a apresentar o tipo extratensivo, adolescentes o intratensivo e o tipo ambigual costuma intensificar em fases avançadas da idade cronológica.

Este resultado mostra mais um indicador importante de ser analisado nas avaliações para manuseio de arma de fogo por trazer informações de como o ambiente está mobilizando o mundo interno do candidato, provocando reações afetivoemocionais, com as mais variadas repercussões na estrutura e funcionalidade da personalidade.

Já sobre a vivência interna e sua relação com a idade é possível perceber na tabela 3 que os indivíduos mais velhos, apresentaram maiores scores no tipo de ressonância "Coartado" assim como o G2 que sugere condições adequadas para lidar com as condições emocionais. Neste caso pode-se inferir que em função da maturidade o sujeito possa demonstrar de forma adequada esse equilíbrio entre o mundo interno e externo.

A partir dos resultados apresentados pode-se constatar que tanto indicadores favoráveis quanto desfavoráveis estão presentes nos dois grupos, mas há uma diferença perceptível entre eles. Entre os indicadores favoráveis ao porte de arma de fogo, eles foram mais frequentes no G2 (não porte). Quanto aos indicadores desfavoráveis, a frequência foi maior no G1 (porte) mostrando a importância de se analisar esses construtos na avaliação para manuseio de arma de fogo.

Os resultados alcançados neste estudo demonstraram que o $\mathrm{Z}$ teste é um instrumento que atende às demandas da avaliação psicológica para porte de arma de fogo e consegue revelar indicadores importantes para a análise do psicólogo credenciado pela Polícia Federal. O Z teste é um instrumento que permite a aplicação em tempo breve, podendo ser aplicado de forma coletiva reduzindo o tempo utilizado na avaliação e permitindo que o psicólogo utilize melhor seu tempo.

Um outro dado importante é a riqueza de informações que o teste traz sobre os construtos da personalidade. $\mathrm{O}$ psicólogo pode aprofundar suas análises até onde for necessário na avaliação que está fazendo, pois o teste permite não só as análises dos determinantes em si, mas também as análises combinadas entre eles. É possível citar como exemplo uma ausência de FM combinada com baixo $\mathrm{F}$ e relação $\mathrm{CF}+\mathrm{C}>\mathrm{F}$ que é sugestivo de compulsão.

A partir da análise dos resultados pode-se então, afirmar que o $\mathrm{Z}$ teste é eficiente e deve ser incluído na bateria de avaliação psicológica para manuseio de arma de fogo.

Importante ressaltar que os resultados da avaliação devem ser pautados no conjunto das técnicas utilizadas no processo o que inclui a entrevista psicológica, pois os construtos psicológicos quando relevantes surgem de forma repetida nos diversos instrumentos utilizados.

\section{Considerações Finais}

De forma geral, esse estudo teve como objetivo principal verificar indicadores do $\mathrm{Z}$ teste para auxiliar na tomada de decisão nas avaliações psicológicas para manuseio de arma de fogo através da comparação de dois grupos de sujeitos: aqueles que se submeteram a avaliação psicológica para manuseio de arma de fogo e um grupo controle com sujeitos que não se submeteram.

Estudar de forma científica características de personalidade para pessoas que farão uso de arma de fogo a partir de uma técnica projetiva é desafiador pela própria natureza do instrumento. Algumas das críticas que as técnicas projetivas recebem dizem respeito à subjetividade de sua interpretação e ao desafio de assegurar a qualidade psicométrica. 
Villemor-Amaral (2009), sinaliza que a análise e interpretação das técnicas chamadas tradicionalmente de projetivas se apoiam mais em pressupostos teóricos do que em evidências originadas em procedimentos psicométricos. Entretanto, para atender as demandas científicas atuais se faz necessário investigar e explorar os resultados de técnicas projetivas também do ponto de vista psicométrico.

$\mathrm{O} \mathrm{Z}$ teste é um instrumento que avalia a personalidade, foi criado com base metodológica no Psicodiagnóstico de Rorschach e destina-se à avaliação psicológica geral e especificamente aos construtos teóricos básicos. Se tratando de avaliação psicológica para manuseio de arma o $\mathrm{Z}$ teste demonstrou sensibilidade para diferenciar alguns aspectos da personalidade dos dois grupos deste estudo e evidenciou que indicadores que são importantes de serem levados em consideração na avaliação psicológica nesse contexto, principalmente os relacionados a cor, controle da expressão emocional $(\mathrm{FC}>\mathrm{CF}+\mathrm{C})$ e o tipo de ressonância interna.

A hipótese levantada foi a de que os indicadores como impulsividade, controle e tendência a excitabilidade emocional pudessem servir nas avaliações como indicadores confiáveis. Esperava-se que tais indicadores surgissem no G1 (porte) e não no G2 (não porte). Observou-se que isso ocorreu em relação aos indicadores que correspondem às respostas de cor. Outros indicadores não se apresentaram relevantes no resultado deste estudo.

Isso pode estar relacionado ao fato de existir características de personalidade semelhantes entre os indivíduos mesmo que em situações diversas, ou ainda que aqueles que não se submeteram à avaliação psicológica para manuseio de arma de fogo possam ter vontade de realizar a avaliação e assim possuem perfil semelhante aos que já se submeteram a esse tipo de avaliação.

$\mathrm{O}$ indicador mais significativo na diferenciação dos dois grupos foi a relação $(\mathrm{FC}>\mathrm{CF}+\mathrm{C})$ que indica o parâmetro ideal indicativo de relacionamento afetivo e emocional com as demais pessoas de forma adequada que aparece na sua forma inversa $(\mathrm{C}+\mathrm{CF}>\mathrm{FC})$ no $\mathrm{G} 1$ (porte).

Ressalta-se que um teste não é valido por si só, por mais robusta que seja a validade teórica do instrumento e dados de validade em outros contextos, mas deve apresentar evidências de validade para as inferências feitas com base nos seus resultados e o mais importante que isso é considerar sua validade relacionada ao contexto em que se pretende utilizar e de fidedignidade dos seus resultados.

Portanto, considera-se que alguns indicadores do $\mathrm{Z}$ teste são importantes para a bateria de testes para avaliação psicológica de manuseio de arma de fogo, porém essa análise deve ser, também, qualitativa, abordando outros instrumentos na aplicação e contexto da própria avaliação. Com isso, considerar o local desta, os sujeitos envolvidos, o momento de vida do avaliado e particularidades pertinentes a cada situação avaliativa, contribuiria para compreensão de possíveis contradições e análise dos dados em seu conjunto.

Em específico para as avaliações para manuseio de arma de fogo se faz necessário a continuação de realização de pesquisas para seja possível haver um maior respaldo teórico, técnico e ético. Só assim será possível delinear um perfil de aspectos favoráveis e não favoráveis que possa sustentar resultados de indicação e contraindicação ao manuseio de arma de fogo; por exemplo, em estudos futuros seria interessante considerar à avaliação de porte de arma para homens e mulheres, pois, existe uma quantidade de mulheres com atividades laborativas que fazem uso de arma de fogo, também, seria importante a realização de estudos transculturais sobre tal fenômeno. Por fim, reforça-se a necessidade de tomada de decisão por parte do profissional psicólogo ser pautada no rigor ético e técnico, pois de sua decisão o porte de arma de fogo poderá passar de um instrumento de defesa e de proteção para um risco de vida de quem a manuseia ou dos que com ele convivem. 


\section{Referências}

Armstrong, J. S., \& Overton, T. S. (1977). Estimating non response bias in mail surveys. Journal Marketing Research, 14, 396-402 http://dx.doi.org/10.2307/3150783.

Brasil (1197). Lei 9.437, de 20 de fevereiro de 1997. Institui o Sistema Nacional de Armas - SINARM, estabelece condições para o registro e para o porte de arma de fogo, define crimes e dá outras providências. Publicada em 21 de fevereiro de 1964. Diário Oficial da União. https://www.planalto.gov.br/ccivil_03/leis/19437.htm

Brasil (2003). Lei 10.826, de 23 de dezembro de 2003. Estatuto do desarmamento. Dispõe sobre registro, posse e comercialização de armas de fogo e munição, sobre o Sistema Nacional de Armas - SINARM, define crimes e dá outras providências. Publicada em 23 de dezembro de 2003. Diário Oficial da União. http://www.planalto.gov.br/cciviL_03/leis/2003/L10.826.htm\#art36

Brasil (2004). Decreto 5.123, de 01 de julho de 2004. Regulamenta a Lei $n^{\circ} 10.826$, de 22 de dezembro de 2003, que dispõe sobre registro, posse e comercialização de armas de fogo e munição, sobre o Sistema Nacional de Armas - SINARM e define crimes. Publicado em 02 de julho de 2004. Diário Oficial da União. http://www.planalto.gov.br/ccivil_03/_Ato20042006/2004/Decreto/D5123.htm

Brasil, Polícia Federal (2014). Instrução normativa no.78/2014-DG/DPF de 10 de fevereiro de 2014. Estabelece procedimentos para o credenciamento e fiscalização de psicólogos responsáveis pela expedição de comprovante de aptidão psicológica para o manuseio de arma de fogo e regulamenta a atuação do psicólogo na avaliação psicológica do vigilante. http://www.pf.gov.br/servicos-pf/armas/credenciamento-psicologos/psicologos-crediciados/IN\%2078-2014.docx/view

Brasil, Polícia Federal. (2020). Sistema Nacional de Armas - SINARM. http://www.dpf.gov.br/servicos/armas/

Cohen, J. (1992a). A power primer. Psychological Bulletin, 112(1), 155.

Cohen, J. (1992b). Statistical power analysis. Current Directions in Psychological Science, 1(3), 98-101.

Conselho Federal de Psicologia (2013). Cartilha Avaliação Psicológica. http://satepsi.cfp.org.br/docs/cartilha.pdf

Cumming, G. (2012). Understanding the new statistics: Effect sizes, confidence intervals, and meta-analysis. Routledge.

Faul F., Erdfelder E., Lang, A., \& Buchner, A. (2007). G*Power 3: A flexible statistical power analysis program for the social, behavioral, and biomedical sciences. Behavior Research Methods, 39, 175-191. 10.3758/BF03193146

Ipea. Instituto de Pesquisa e Economia aplicada (2020). Atlas da violência. https://www.ipea.gov.br/atlasviolencia/ publicacoes/51/atlas-da-violencia-2020

Lenhard, E, \& Lenhard A. (2016). Calculation of effect sizes. Psychometrica. 10.13140/RG.2.1.3478.4245

Nascimento, D. C., Tibana R. A., Ferreira, G. M., \& Prestes, J. (2014) Testes de normalidade em análises estatísticas: uma orientação para praticantes em ciências da saúde e atividade física. Revista Mackenzie de Educação Física e Esporte, 14(2), 73-77.

Oliveira, A. L. S., Luna, C. F. \& Silva, M. G. P. (2020). Homicídios do Brasil na última década: uma revisão integrativa. Ciência \& Saúde Coletiva, 25(5), 1925-1934. https://doi.org/10.1590/1413-81232020255.09932018

Prodanov, C. C. (2013). Metodologia do trabalho científico [recurso eletrônico]: métodos e técnicas da pesquisa e do trabalho acadêmico. (2. ed.) - Novo Hamburgo: Feevale. http://www.feevale.br/Comum/midias/8807f05a-14d0-4d5b-b1ad1538f3aef538/E-book\%20Metodologia\%20do\%20Trabalho\%20Cientifico.pdf

Resende, M. A. (2017). Avaliação psicológica para concessão do porte de arma de fogo à população e policiais da PMMG. Psicologia e saúde mental. 3(6), 113-131. http://revista.policiamilitar.mg.gov.br/periodicos/index.php/psico logia/article/view/97/195

Resende, M. A. (2019). Desafios da avaliação psicológica para porte de arma de fogo. Revista Psicologia e suas conexões.1(2). http://revistaadmmade.estacio.br/index.php/psicologiaesuasconexoes/article/viewPDFInterstitial/6681/47965702

Tabachnick, B. G., \& Fidell, L. S. (2001). Using Multivariate Statistics. (4a ed.), Allyn and Bacon. 
Vaz, C. E., \& Alchieri, J. C. (2016). Z teste: coletivo e individual: técnica de Zulliger. (3a ed.). Hogrefe CETEPP.

Villemor-Amaral, A. E. (2009). Métodos Projetivos em Avaliações Compulsórias: indicadores e perfis. In: C. S. Hutz, Avanços e polêmicas em avaliação psicológica (157-174). Casa do Psicólogo.

Villemor-Amaral, A. E., \& Machado, M. S.(2011). Indicadores de depressão do Zulliger no Sistema Compreensivo (ZSC). Paidéia (Ribeirão Preto), 21(48), 21-27. https://dx.doi.org/10.1590/S0103-863X2011000100004 